\title{
In pursuit of COVID-19 surgical risk stratification to manage a limited workforce and supplies in minimally invasive surgery
}

\author{
Kryspin Mitura ${ }^{1}$, Piotr Myśliwiec ${ }^{2}$, Wojciech Rogula ${ }^{3}$, Michał Solecki ${ }^{4}$, Jarosław P. Furtak ${ }^{5}$, Michał Kazanowski ${ }^{6}$, \\ Stanisław Kłęk ${ }^{7}$, Michał Nowakowski ${ }^{8}$, Michał Pędziwiatr ${ }^{8}$, Marek Zawadzki ${ }^{9}$, Grzegorz Wallner ${ }^{4}$, Jacek Sobocki ${ }^{10}$ \\ ${ }^{1}$ Faculty of Medical and Health Sciences, University of Natural Sciences and Humanities, Siedlce, Poland \\ ${ }^{2} 1^{\text {st }}$ Department of General and Endocrine Surgery, Medical University of Bialystok, Bialystok, Poland \\ ${ }^{3}$ Department of Paediatric Surgery, University Paediatric Hospital, Jagiellonian University Medical College, Krakow, Poland \\ ${ }^{4} 2^{\text {nd }}$ Department of General, Gastrointestinal, and Surgical Oncology, Alimentary Tract, Medical University of Lublin, Lublin, Poland \\ ${ }^{5}$ Department of General Surgery, Pulawy Hospital, Pulawy, Poland \\ ${ }^{6}$ Lower Silesian Oncology Centre in Wroclaw, Wroclaw, Poland \\ ${ }^{7}$ Department of General Surgery, Stanley Dudrick Memorial Hospital, Krakow, Poland \\ ${ }^{8} 2^{\text {nd }}$ Department of General Surgery, Jagiellonian University Medical College, Krakow, Poland \\ ${ }^{9}$ Regional Specialist Hospital, Faculty of Health Sciences, Wroclaw Medical University, Wroclaw, Poland \\ ${ }^{10}$ Department of General Surgery and Clinical Nutrition, Medical Centre of Postgraduate Education, Warsaw, Poland
}

Videosurgery Miniinv 2020; 15 (3): 416-423

DOI: https://doi.org/10.5114/wiitm.2020.95073

\begin{abstract}
The protective barriers used so far in surgery do not provide adequate protection against SARS-COV-2 virus, and reinforced protective equipment is needed. The rapid increase in the number of patients and the worldwide panic associated with the increasingly low availability of protective equipment has resulted in a shortage of protective equipment in many hospitals. Appropriatepersonal protective equipment must be provided so that the surgical team proceeding to surgery is not excluded from the further struggle for patients' health, especially in MIS. Reckless and excessive use of maximum protective equipment may result in a severe shortage of these products when the number of infected persons requiring surgery increases. The use of a structured infection risk scheme for medical staff, depending on the results of reverse transcription polymerase chain reaction assays and COVID-19 symptoms, combined with the division of protection equipment into three groups, allows easy selection of an appropriate clothing scheme for the clinical setting.
\end{abstract}

Key words: COVID-19, safety, personal protective equipment, FFP3, pandemic, epidemic.

\section{Introduction}

The rapid spread of SARS-CoV-2 infection around the world has shaken the existing health care systems to its very core. Patients who may be potentially infected report to hospitals for surgical treatment; thus, they pose a risk of spread of the virus - both to other patients and to medical personnel [1]. This has resulted in the introduction of restrictions on elec- tive surgeries and postponement of majority of procedures [2]. In addition, the protective barriers used so far in surgery do not provide adequate protection against spread of the virus. Hence, reinforced protective equipment is needed [3]. The rapid increase in the number of infected patients and the worldwide panic has resulted in a shortage of protective equipment in many hospitals. The media worldwide report dramatic images of medical personnel dressed in homemade

\section{Address for correspondence}

Kryspin Mitura MD, PhD, Faculty of Medical and Health Sciences, University of Natural Sciences and Humanities, Siedlce, Poland,

phone: +48 602809 035, e-mail: chirurgia.siedlce@gmail.com 
protective aprons made of plastic garbage bags and self-constructed surgical masks instead of professional personal protective equipment (PPE) $[4,5]$.

Furthermore, the lack of evidence for determining the safety of various surgical accesses has led to the spread of myths, which causes confusion among surgeons. Both recommendations against minimally invasive surgery (MIS) and those in favour of this method are available [6-9].

Surgery is a unique branch of medicine, wherein a trained professional cannot be replaced by another specialist [10]. Lack of proper surgical care may cause diseases that are currently considered trivial to become dangerous. Therefore, the safety of the surgical team is of utmost importance. During the pandemic, surgeons must be ready for interactions with SARS-CoV-2 patients. However, appropriate PPE must also be provided so that the surgical team is not excluded from the further struggle for patients' health. The current equipment resources are limited by sudden shortages. Therefore, the rational use of these resources is of great importance. Considering both the limited availability of rapid diagnostic tests for SARS-CoV-2, the possibility of false negative results, the asymptomatic course of COVID-19 disease in many patients, and the incubation period, it is important to determine the current immune status of patients undergoing urgent surgery $[11,12]$. Lack of knowledge of who is infected may:

- cause adverse outcomes in "COVID-19 asymptom-

atic" patients undergoing surgery,

- compromise the health of other patients,

- expose health care providers [13].

On the other hand, the need to isolate patients with unknown SARS-CoV-2 status results in unnecessary use of PPE. The aim of this publication is to determine the method of surgical risk stratification depending on the available objective data concerning both symptoms of the disease and the results of additional tests.

\section{Perioperative risk of infection transmission}

The classifications for risk assessment currently used in surgery mainly concern the possibility of complications in patients undergoing surgery $[13,14]$. However, the literature lacks universally validated methods to assess the risk of infection for surgeons during surgery. Before the COVID-19 pandemic, the main concern of surgeons was to avoid blood-borne infection. Therefore, the use of an apron, eye protection, and gloves was considered to be sufficient [15]. In addition, it was recommended that surgeons with present cuts on their hands avoid participation in surgery. In the case of an accidental cut during surgery, appropriate protocols (developed in each hospital) were introduced. These actions were primarily aimed at avoiding HIV infection as well as hepatitis $B$ and C (HBV, HCV) [16]. The current COVID-19 pandemic, however, has drawn attention to the danger of SARS-CoV-2 transmission through the droplet/airborne route of infection. It should be noted that such transmission occurs most often during intubation of the patient [17]. Therefore, during intubation, only necessary persons should be present in the operating room; the surgical team should enter the operating room only after completion of the intubation and should leave the operating room before extubation.

It is unclear whether the virus particles can be released from the surgical field, both from blood and smoke originating from electrocoagulation of tissues [18]. This assumption is based on the fact that during laser coagulation of pathological lesions, particles of the human papillomavirus (HPV) originating from patients were found both on the mucosae and conjunctivae of a treating surgeon and on filters of smoke extraction systems from the surgical field [19]. It should be noted that in the case of viruses spreading through the droplet route of infection, the aerosol generated in the air during surgery, entering the ocular conjunctiva, can be transmitted to the nasal mucosa and paranasal sinuses through the tear ducts [20]. The presence of RNA virus has also been confirmed in the gastrointestinal tract of patients; therefore, small and large intestine surgeries are also classified as high-risk surgical procedures [21]. However, until evidence becomes available, it must be assumed that such a risk exists.

Working in a potentially infected environment, such as the operating room during surgery on a COVID-19-positive patient, causes virus particles in the air to settle on various surfaces, including uncovered skin and staff clothing. There are many reports about the potential for long-term persistence of SARS-CoV-2 virus on various surfaces [22]. Therefore, when performing these surgical procedures, the risk of contamination of the uncovered skin (e.g. neck and neckline) of the personnel should also be considered - from the skin, the virus particles may be ac- 
cidentally transmitted to the mucosa or conjunctivae of the medical staff. For this reason, such body parts also need to be protected during surgeries.

\section{Risk stratification for the infection during surgery}

Guo et al. noted that after the COVID-19 outbreak in Wuhan, China a significant discrepancy in the percentage of infected orthopaedic surgeons was found (1.5\% to $20.7 \%)$ [1]. The article emphasises that participation in live trainings on the use of PPE showed a protective effect against COVID-19 (odds ratio $(\mathrm{OR})=0.12$ ). It was highlighted that not using N95 masks was a significant risk factor $(\mathrm{OR}=5.20,95 \%$ confidence interval (CI): 1.09-25.00), while non-stop mask-wearing showed a protective effect $(\mathrm{OR}=0.15)$. At the same time, extreme fatigue and exhaustion of the medical staff were also risk factors for surgical infection $(\mathrm{OR}=4,95 \% \mathrm{Cl}$ : 1-16). Therefore, it is important to introduce all possible forms of protection to save human resources (workforce) and not to overburden those healthcare workers who continue to work with patients with duties, especially in countries where there is a shortage of surgeons. At the same time, the lack of sufficient supplies of PPE means that surgeons often have to make their own choices as to which form of protection should they select for an operation, so that the available resources are not used too early. Serological tests are performed to identify infected patients or those suspected of being infected. The ELISA with virtual nucleoproteins detects IgM and IgG antibodies [23]. The reverse transcription polymerase chain reaction (RT-PCR) assay allows the detection of even a very small amount of SARS-CoV-2 enzyme. However, in the first days after infection, its efficacy is only $50 \%$ in nasopharyngeal swabs and
$25 \%$ in rectal swabs [24]. In their analysis, Zhang et al. noted that the level of antibodies in the first days after the infection may be too low to be found in the assay; therefore, the operated patient with persistent infection may still have a negative SARSCoV-2 test result [25]. Failure to properly assess the presence or absence of the virus in the body results in false positive or negative results. The numerous causes contributing to false results include incorrect material collection, contamination of the collected material, collection from the wrong region (throat instead of nasopharynx), testing too early, the use of an inadequate test, incorrect sample dilution, assay performed by an inexperienced worker, and the possibility of a secondary infection or laboratory error [25].

Taking into account the RT-PCR SARS-CoV-2 result and the presence of disease symptoms, we divided patients into four risk groups with regard to the infection of others while performing surgery in this patient group (Table I) [26].

\section{Division of available PPE}

Due to the increase in the number of COVID-19 cases in Poland (more than ten thousands cases until Apr 22, 2020), and the consequent increase in the number of asymptomatic patients who may already be infected without any significant epidemiological history, the following PPE are used: a surgical mask and a cap for an operated patient; N95 FFP3 mask along with surgical mask, a cap or bonnet, protective goggles or glasses, a face shield, a waterproof barrier surgical gown (possible coverall protective suit), surgical gloves, and protective footwear (covered, short shoes are permitted). Personal protective equipment is required for each 'close contact', which includes surgical operations, as well as other medical procedures taking place in the operating room, e.g.

Table I. Patient groups according to the risk of infecting others [26]

\begin{tabular}{|lccc|}
\hline $\begin{array}{l}\text { Risk group for the infection } \\
\text { of others }\end{array}$ & COVID-19 symptoms & SARS-CoV-2 test & In-hospital treatment \\
\hline Low & None & Negative & Standard \\
\hline Moderate & None & Pending & Standard \\
\hline High & None & Positive & Specialist \\
\cline { 2 - 4 } & Present & Negative & \\
\cline { 2 - 4 } & Present & Pending & Specialist \\
\hline Very high & Present & Positive & \\
\hline
\end{tabular}


intubation, local anaesthesia, and intravenous access insertion. It should be noted that an increased risk of SARS-CoV-2 infection is also present during endoscopic examinations and procedures, especially in the upper gastrointestinal tract $[26,27]$.

A surgical mask is designed to reduce the emission of aerosol exhaled from the airways containing virus particles. For the N95 mask, the letter N indicates that the product is not resistant to oil. The number 95 (in N95) indicates the degree of filtration of particles in the air (95\%). The phrase FFP (in FFP3) means 'filtering facepiece', in other words: 'filtering half masks'. FFP2 or FFP3 (also FFP1) is a class/level of filtration. FFP2 captures at least $94 \%$ of particles up to $0.6 \mu \mathrm{m}$, whereas FFP3 captures at least $99 \%$ of particles up to $0.6 \mu \mathrm{m}$. Valve-less masks are preferred because a valved mask does not protect the patient from operator pathogens. If only valved masks are available, an ordinary surgical mask should be worn on top of such a mask. For ease of use, we always recommend wearing a surgical mask on the mask. According to the manufacturers' instructions for use, FFP2 masks do not protect against viruses transmitted through the droplet route of infection [26].

When wearing a coverall protective suit, a surgical cap is not required. When wearing a surgical gown, two caps can be used (the second for goggles and mask straps). In case of lack of a coverall protective suit, it is recommended that an astro cap be worn (it covers the neckline and neck) and an additional neckerchief. Surgical goggles with an elastic strap or temple eyeglasses should be worn to cover the orbital region. If it is necessary to use corrective lenses, goggles should be placed on top of existing glasses (corrective lenses and glasses should be decontaminated like all other equipment) [26]. The risk of contact lenses being displaced or falling out during surgery (without the possibility of correcting their position with PPE used) should be taken into account in persons wearing contact lenses. There is currently no scientific evidence that contact lenses should be not used, but they are not recommended [28]. A face shield protects the facial area against droplets. It is head-mounted, adjustable at the back of a clasp. A disposable biological protection suit with a hood tightly surrounds the face and chin. Orange (reusable) protection suits are designed for emergency service teams, and they are not adapted to indoor work for a long time and under the influence of increased temperature (operating lights, additional sterile apron on top of the suit). White barrier coveralls are preferred. It is advisable to wear a coverall protective suit that is at least one size larger because it facilitates putting on and taking off the suit. In the case of wearing highly protective rubber boots, we choose a model of the protection suit without "feet" (suit leg outside the rubber boot). When wearing short footwear, a coverall protective suit with integrated foot protectors is recommended [26]. Some coverall protective suits have separate high foot protectors tied at the shin level. Such a solution is an alternative to rubber boots.

In the absence of a coverall protective suit, a double sterile barrier surgical gown (class 4), in accordance with EN 13795-1 standard, can be used (or vice versa). If there are no barrier coveralls, a foil apron underneath the coverall protective suit can be used. Double surgical gloves are also used. The first pair of gloves - designed for the sleeves of the coverall protective suit or inner apron - is longer, covering at least $1 / 3$ of the forearm (extended gloves used in gynaecology), and a second pair of gloves is designed for the surgical gown. Protective footwear should cover fully, i.e. without any holes or perforated surfaces. The best footwear is plain, extended rubber boots (wellingtons). Plastic shoe covers can be used on the outside of the shoes.

Depending on the combinations applied for protective equipment, PPE sets can be divided into three groups, which are listed in Table II [26].

At present, during the ongoing COVID-19 pandemic, it is still not possible to state with absolute certainty that the operated patient, who belongs to a low-risk group of infection, is in fact uninfected, due to the possibility of obtaining false negative results. For this reason, we recommend the use of enhanced protective equipment, even for patients belonging to a low-risk group of infection (Table III).

\section{Eligibility for surgery}

Before each surgical procedure, the possibility of SARS-CoV-2 infection and its consequences should be discussed with the patient. This information should be included in the informed consent form [26]. Before surgery, it is necessary to perform the SARS-CoV-2 assay if the patient's condition allows them to wait for the test result. However, it should be noted that this waiting time must be as short as 
Table II. Types of personal protective equipment sets [26]

\begin{tabular}{|c|c|c|c|}
\hline $\begin{array}{l}\text { PPE for invasive } \\
\text { procedures }\end{array}$ & Standard & Extended & Maximum \\
\hline Indications & Low risk & Moderate risk & High risk \\
\hline Mask & N95 FFP3* and surgical mask & N95 FFP3 and surgical mask & N95 FFP3 and surgical mask \\
\hline Headwear & Surgical bonnet & $\begin{array}{c}\text { Astro surgical cap with head and } \\
\text { neck protection }\end{array}$ & Waterproof cap or hood \\
\hline Eye protection & Safety glasses/goggles & $\begin{array}{c}\text { Safety glasses/goggles and face } \\
\text { shield }\end{array}$ & $\begin{array}{c}\text { Safety glasses/goggles and face } \\
\text { shield }\end{array}$ \\
\hline Body protection & Barrier surgical gown & Double-barrier surgical gown & $\begin{array}{l}\text { One-piece biological protection } \\
\text { suit and surgical gown }\end{array}$ \\
\hline Hand protection & $\begin{array}{c}\text { Disposable gloves consider } \\
\text { double gloves }\end{array}$ & Double $(\times 2)$ disposable gloves & Triple $(\times 3)$ disposable gloves \\
\hline Leg protection & Footwear protection & Long safety footwear & $\begin{array}{c}\text { Long safety footwear if the } \\
\text { protection suit does not protect } \\
\text { your feet }\end{array}$ \\
\hline
\end{tabular}

*In patients operated on under accelerated conditions after 1 week of isolation with negative RT-PCR results and chest CT scans, a lower degree of protection is exceptionally allowed when FFP3 masks are not available (e.g. FFP2).

Table III. Risk of contamination of medical personnel during surgeries and required personal protective equipment (PPE)

\begin{tabular}{|c|c|c|c|}
\hline \multirow{2}{*}{$\begin{array}{l}\text { COVID-19 } \\
\text { symptoms }\end{array}$} & \multicolumn{3}{|c|}{ RT-PCR SARS-CoV-2 } \\
\hline & Negative & Unknown & Positive \\
\hline \multirow[t]{2}{*}{ No } & LOW RISK & MODERATE RISK & HIGH RISK \\
\hline & enhanced PPI & enhanced PPI & maximum PPI \\
\hline \multirow[t]{2}{*}{ Yes } & HIGH RISK & HIGH RISK & VERY HIGH RISK \\
\hline & maximum PPI & maximum PPI & maximum PPI \\
\hline
\end{tabular}

possible. In cases of emergency and urgent surgical procedures, i.e. when rapid immune tests or RT-PCR assays are not possible, it should be assumed that every operated patient is infected with SARS-CoV-2. Only patients with a negative epidemiological history, who were tested with RT-PCR assay, may constitute a lower risk group (early stage of infection, asymptomatic patients). This assumption is based on the rapid growth of new infections and the need to protect medical personnel [26]. The use of an epidemiological history, immune RT-PCR assays, or previous chest CT scans/X-rays can be used to assess risk. In the case of A\&E CT of the abdominal cavity, the examination is extended to include the chest CT scan [29]. In cases where non-operative treatment is also possible (uncomplicated acute cholecystitis, Hinchey I-II diverticulitis, etc.), conservative therapy should be provided (Figure 1).

\section{MIS vs. open approach}

Despite the evident advantages of minimally invasive surgery, during the COVID-19 pandemic the selection of an optimal surgical technique is one of the most common clinical questions posed by surgeons [26, 30]. At present, we do not have any clear evidence of the relative risk of laparoscopy towards laparotomy (open surgery) in patients infected with SARS-CoV-2 (Table IV) [31]. Although research on HBV viruses has shown that laparoscopy may lead to the formation of an aerosol containing virus particles from the patient's blood, there is no clear evidence that there is a similar effect in the case of coronavirus, and in particular, that patients with SARS-CoV-2 infection do not have the virus in their blood [18, 26], especially given the fact that there is no evidence that such an effect could be associated with 


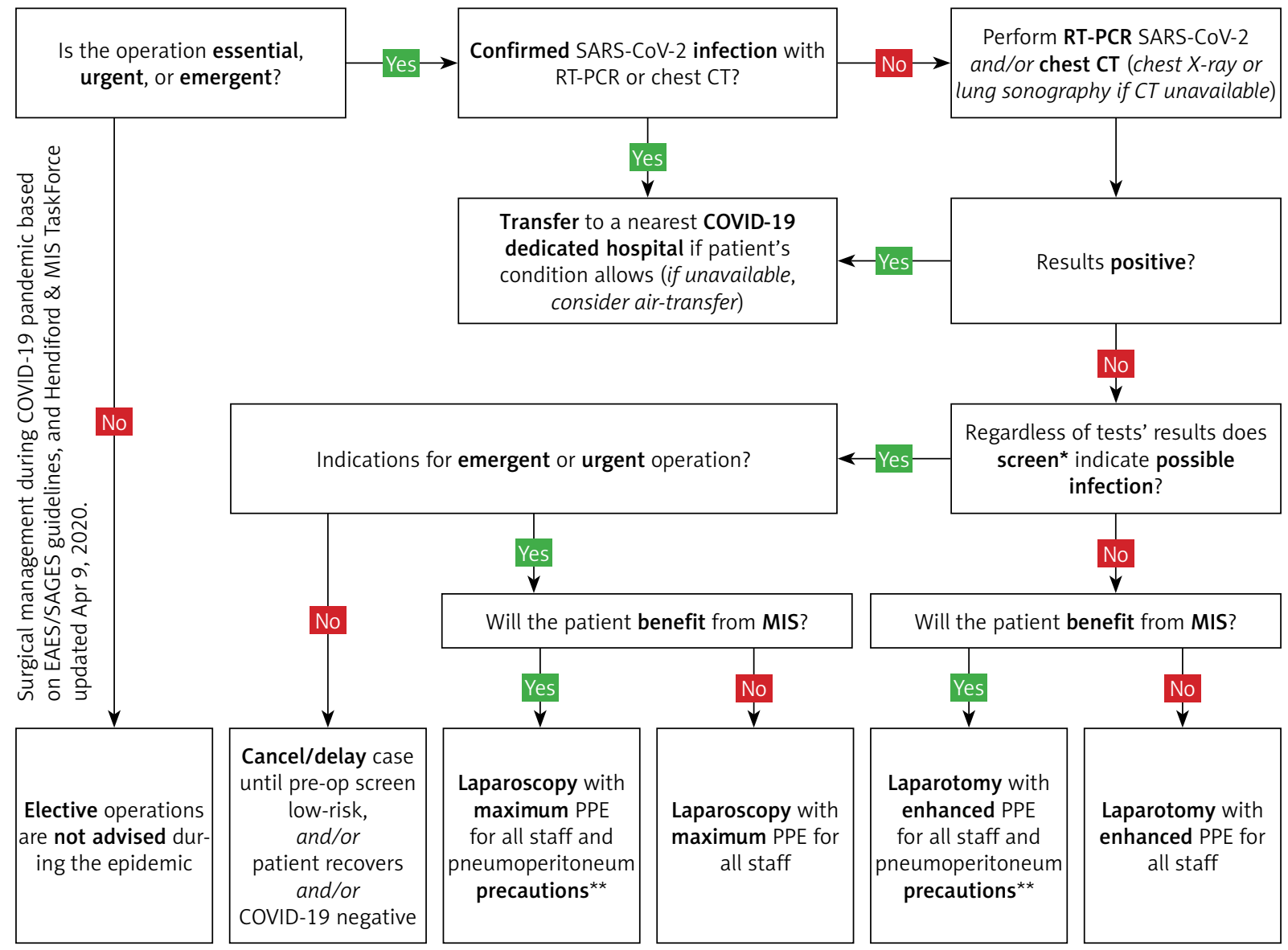

*History, physical examination, and a patient questionnaire, which is directed at flu-related symptoms (fever, dyspnea, cough, ansomnia), travel, and exposure, non-contrast chest CT scan.

**Lowest possible pneumoperitoneum pressure (10-12 $\mathrm{mm} \mathrm{Hg}$ ); no plume of gas to escape; desuflation via suction device; do not vent into the room; use insuflation-filtration device (i.e. AirSeal), if available.

Figure 1. Management algorithm for surgery during the COVID-19 pandemic

Table IV. Summary of guidelines of international surgical societies in the assessment for the surgical access technique applied during the COVID-19 pandemic [6-9]

\begin{tabular}{|lcccc|}
\hline Publication date & \multicolumn{1}{c|}{ Origin of the guidelines } & \multicolumn{2}{c|}{ Evaluation of benefits from the technique used } \\
\hline Mar 26, 2020 & Royal College of Surgeons of England & Laparoscopy & $<$ & Laparotomy \\
\hline Mar 29, 2020 & $\begin{array}{c}\text { Society of American Gastrointestinal and } \\
\text { Endoscopic Surgeons (SAGES) }\end{array}$ & Laparoscopy & $=$ & Laparotomy \\
\hline Mar 30, 2020 & $\begin{array}{c}\text { European Association for Endoscopic } \\
\text { Surgeons (EAES) }\end{array}$ & Laparoscopy & $=$ & Laparotomy \\
\hline Apr 4, 2020 & Spanish Association of Surgeons & Laparoscopy & $>$ & Laparotomy \\
\hline
\end{tabular}

minimally invasive procedures only. On the basis of the assumption that SARS-CoV-2 virus might be released in the form of an aerosol in carbon dioxide used to create pneumoperitoneum, Di Saverio et al. have concluded that the decision on the use of laparoscopy should be made individually, i.e. with refer- ence to the patient, their disease, and the surgeon's experience, and available safety systems (AirSeal gas evacuation system or gasless laparoscopy) [32]. At the same time, the authors have emphasised that laparoscopy, performed in the enclosed space of the abdominal cavity, can reduce the spread of aerosol 
when compared to open surgery, when preserving the (wall) competence of the abdominal system and the removal of carbon dioxide from the abdominal cavity using a vacuum aspirator. Mintz et al. [33] presented a non-expensive and effective method of removing gas from the peritoneal cavity using a filter applied for ventilators (respirators); they stressed that laparoscopy may be the recommended method because during traditional surgery there is no possibility of full control over the smoke released during the use of electrosurgical instruments.

Therefore, in view of the availability of non-expensive and effective methods of purifying the gas used for laparoscopy, it is emphasised that purifying the air from particles contained in aerosols can be much more difficult during open surgery [26] In addition, laparoscopy creates a natural physical barrier between medical personnel and the potential source of infection, thus reducing the surgical team's exposure to the patient's body fluids and the risk of occupational exposure. Furthermore, one should also remember the well-documented advantages of MIS (which cannot be overestimated in epidemic conditions), such as a reduced number of complications (including pneumonia), reduced mortality (which may be particularly significant for patients with COVID-19) and shortened period of hospitalisation [26, 34].

Considering the data presented above, during laparoscopic surgeries, the following is recommended: - to preoperatively check the proper functioning of laparoscopic equipment (trocars, seals, etc.);

- to appropriately plan and make the smallest possible incisions for the insertion of trocars, providing tightness around them;

- to maintain intra-abdominal pressure of 8-11 $\mathrm{mm} \mathrm{Hg}$;

- to evacuate smoke during surgery through a suction unit connected to the central vacuum system and/or to use smoke filters (for this purpose, respirator filters connected via an adapter may also be used);

- to perform desufflation, using the techniques described above, before making an additional incision (e.g. minilaparotomy);

- to avoid prolonged keeping of the patient in the Trendelenburg position.

Therefore, when taking precautions regarding pneumoperitoneum, traditional and laparoscopic surgeries may be performed during the COVID-19 pandemic. The surgical technique should be individ- ually adapted to the patient, their disease requiring the treatment and the operator's experience [26, 35]. Operating rooms intended for patients with suspected or confirmed SARS-CoV-2 infection should be appropriately filtered and ventilated. If possible, these rooms should be dedicated exclusively to this group of patients, separated from those used for other urgent surgeries. If available, pressurised rooms should be used. The number of medical personnel present in the operating room should be limited to an absolute minimum. If possible, the surgery should be performed by an experienced surgeon. Due to the expected ever-growing number of asymptomatic patients who will require surgical intervention, the abovementioned principles should be applied in each case to reduce the possibility of infection within surgical teams.

\section{Summary}

Risk stratification for the infection of the surgical team is necessary to establish PPE management during the COVID-19 pandemic. Reckless and excessive use of the maximum protective equipment may result in a severe shortage of these products when the number of infected persons requiring surgical treatment increases. On the other hand, failure to use adequate protective equipment when performing surgery on an infected patient exposes the entire surgical team to infection and may exclude professionals from the healthcare system. At the same time, there are reports on the efficacy of various provisional solutions to extend the shelf life or reuse of products previously considered to be disposable, such as N95 FFP3 masks, which are an essential element of protection in the case of airborne SARS-CoV-2 virus transmitted through the droplet route of infection.

The use of a structured infection risk scheme for medical staff, depending on the results of RT-PCR assays and COVID-19 symptoms, combined with the division of protection equipment into three protection groups, allows quick and easy selection of an appropriate clothing scheme for the clinical setting. The scheme presented by us is based on the authors' own observations and experience but requires further validation to determine its ultimate clinical significance.

\section{Conflict of interest}

The authors declare no conflict of interest. 


\section{References}

1. Guo X, Wang J, Hu D, et al. Survey of COVID-19 disease among orthopaedic surgeons in Wuhan, People's Republic of China. J Bone Joint Surg Am 2020 Apr 8. doi: 10.2106/JBJS.20.00417.

2. Operational considerations for case management of COVID-19 in health facility and community. WHO. 19 March 2020.

3. Handbook of COVID-19 Prevention and Treatment Compiled According to Clinical Experience. Zheijang University School of Medicine 2020.

4. Bowden E, Campanile C, Golding B. Worker at NYC hospital where nurses wear trash bags as protection dies from coronavirus. New York Post, March 25, 2020.

5. Dwyer K. Should You Use a Bandana or a Scarf as a Face Mask When You Go Out? Women's Health Apr 4, 2020.

6. Updated Intercollegiate General Surgery Guidance on COVID-19. Royal College of Surgeons. Apr 6, 2020. Available at: https://www.rcseng.ac.uk/coronavirus/joint-guidance-for-surgeons-v2/

7. Pryor A. SAGES and EAES recommendations regarding surgical response to COVID-19 crisis. Mar 29, 2020. Available at: https:// www.sages.org/recommendations-surgical-response-covid-19/

8. EAES and SAGES recommendations regarding surgical response to COVID-19 crisis. Mar 30, 2020. Available at: https:// eaes.eu/eaes-and-sages-recommendations-regarding-surgical-response-to-covid-19-crisis/

9. Surgical management of patients with COVID-19 infections. Recommendations of the Spanish Association of Surgeons. Apr 4, 2020. Available at: https://eaes.eu/recomendaciones-de-la-asociacion-espanola-de-cirujanos-in-spanish/1-recomendations-for-perioperative-managment-during-the-pandemic-covid19-v-2/

10. Mitura K, Kozieł S, Komor K. Can the surgeon live his whole life? Analysis of the risk of death related to the profession. Pol Przegl Chir 2018; 90: 18-24.

11. Xiao AT, Tong YX, Zhang S. False-negative of RT-PCR and prolonged nucleic acid conversion in COVID-19: rather than recurrence. J Med Virol 2020 Apr 9. doi: 10.1002/jmv.25855.

12. Wynants L, Van Calster B, Bonten MMJ, et al. Prediction models for diagnosis and prognosis of covid-19 infection: systematic review and critical appraisal. BMJ 2020; 369: m1328.

13. Chand M, Armstrong T, Britton G, Nash GF. How and why do we measure surgical risk? J R Soc Med 2007; 100: 508-12.

14. Bose S, Talmor D. Who is a high-risk surgical patient? Curr Opin Crit Care 2018; 24: 547-53.

15. Moloughney BW. Transmission and postexposure management of bloodborne virus infections in the health care setting where are we now? CMAJ 2001; 165: 445-51.

16. Deuffic-Burban S, Delarocque-Astagneau E, Abiteboul D, et al. Blood-borne viruses in health care workers: prevention and management. J Clin Virol 2011; 52: 4-10.

17. Zou L, Ruan F, Huang M, et al. SARS-CoV-2 viral load in upper respiratory specimens of infected patients. N Engl J Med 2020; 382: 1177-9.

18. Wölfel R, Corman VM, Guggemos W, et al. Virological assessment of hospitalized patients with COVID-2019. Nature 2020 Apr 1. doi: 10.1038/s41586-020-2196-x.
19. Ferenczy A, Bergeron C, Richart RM. Human papillomavirus DNA in $\mathrm{CO} 2$ laser-generated plume of smoke and its consequences to the surgeon. Obstet Gynecol 1990; 75: 114-8.

20. Barrett WL, Garber SM. Surgical smoke: a review of the literature. Is this just a lot of hot air? Surg Endosc 2003; 17: 979-87.

21. Wu Y, Guo C, Tang L, et al. Prolonged presence of SARS-CoV-2 viral RNA in faecal samples. Lancet Gastroenterol Hepatol 2020; 5: 434-5.

22. Walls AC, Park YJ, Tortorici MA, et al. Structure, function, and antigenicity of the SARS-CoV-2 spike glycoprotein. Cell 2020; 181: 281-2.e6.

23. Zhou P, Yang XL, Wang XG, et al. Discovery of a novel coronavirus associated with the recent pneumonia outbreak in humans and its potential bat origin. BioRxiv 2020. doi: https:// doi.org/10.1101/2020.01.22.914952.

24. Wan Z, Zhang YN, He Z, et al. A melting curve-based multiplex RT-qPCR assay for simultaneous detection of four human coronaviruses. Intern J Mol Sci 2016; 17: 1880.

25. Zhang W, Du RH, Li B, et al. Molecular and serological investigation of 2019-nCoV infected patients: implication of multiple shedding routes. Emerg Microb Infect 2020; 9: 386-9.

26. Mitura K, Mysliwiec P, Rogula W, et al. Guidelines for the management of surgical departments in non-uniform hospitals during the COVID-19 pandemic. Pol Przegl Chir 2020; 2: 48-56.

27. Guidance for wearing and removing personal protective equipment in healthcare settings for the care of patients with suspected or confirmed COVID-19. ECDC, Stockholm 2020.

28. Jones L, Walsh K, Willcox M, et al. The COVID-19 pandemic: important considerations for contact lens practitioners. Cont Lens Anterior Eye 2020 https://doi.org/10.1016/j.clae.2020.03.012.

29. Leidraad Preoperatieve diagnostiek naar COVID-19 bij asymptomatische patiënten in afwachting tot chirurgie definitieve versie 02-04-2020. https://www.demedischspecialist.nl

30. Rubinkiewicz M, Pisarska M, Zarzycki P, et al. High compliance to ERAS protocol does not improve overall survival in patients treated for resectable advanced gastric cancer. Videosurgery Miniinv 2020. doi:10.5114/wiitm.2020.92833.

31. Morris SN, Nickles Fader A, Magdy P, et al. Understanding the "Scope" of the problem: why laparoscopy is considered safe during the COVID-19 pandemic. J Minim Invasive Gynecol 2020; 27: 789-91.

32. Di Saverio S, Pata F, Gallo G, et al. Coronavirus pandemic and Colorectal surgery: practical advice based on the Italian experience. Colorectal Dis 2020 Mar 31. doi: 10.1111/codi.15056.

33. Mintz Y, Arezzo A, Boni L, et al. A low cost, safe and effective method for smoke evacuation in laparoscopic surgery for suspected coronavirus patients. Ann Surg 2020 doi: 10.1097/ SLA.0000000000003965.

34. Coccolini F, Catena F, Pisano M, et al. Open versus laparoscopic cholecystectomy in acute cholecystitis. Systematic review and meta-analysis. Int J Surg 2015; 18: 196.

35. Brücher BL, Nigri G, Tinelli A, et al. COVID-19: pandemic surgery guidance. 4open 2020; 3: 1-19.

Received: 20.04.2020, accepted: 28.04.2020. 